\title{
EFEITOS DE UM PROGRAMA REGULAR DE ATIVIDADE GENERALIZADA SOBRE OS DISTÚRBIOS MOTORES DE UMA PESSOA COM DOENÇA DE PARKINSON
}

\author{
Hayka Kattriny Texeira* \\ Gerson Carneiro de Farias** \\ Marcus Fraga Vieira**
}

\begin{abstract}
RESUMO
O objetivo deste estudo foi analisar os efeitos de seis meses de um programa regular de atividade física sobre os distúrbios motores em uma pessoa idosa com a doença de parkinson. O sujeito pesquisado foi uma pessoa idosa do sexo feminino, sedentária, portadora da doença há dez anos. O programa foi desenvolvido durante seis meses com o apoio do Centro Goiano de Neurologia e Neurocirurgia. Para avaliar o nível de rigidez, a bradicinesia, a capacidade de se levantar da cadeira e se locomover e as alterações posturais foi utilizado um exame motor (BRITO, 1998), com avaliações antes e após o programa. Com dois meses de atividade física houve melhora na rigidez, na bradicinesia e no equilíbrio, contribuindo para a autonomia e independência do sujeito pesquisado.
\end{abstract}

PALAVRAS-CHAVE: Doença de Parkinson - Idoso - Atividade física

\section{INTRODUÇÃO}

A rigidez, a bradicinesia (movimentos lentos), os tremores e as alterações posturais são distúrbios motores característicos da doença de parkinson que acomete principalmente pessoas idosas, podendo causar sensíveis alterações motoras e psicológicas com a evolução da doença, contribuindo para a debilidade na capacidade funcional ${ }^{1}$ do idoso e, conseqüentemente, prejudicando sua autonomia e qualidade de vida. ${ }^{2}$

Os problemas físicos são, em grande parte, resultantes da imobilidade pela idade e doença. Uma pessoa idosa ativa, que pratica atividade física além dos movimentos do cotidiano e do trabalho, possivelmente terá menor predisposição para desenvolver complicações clínicas gerais em comparação a uma pessoa idosa inativa

${ }^{*}$ Acadêmica do $4^{\circ}$ ano do curso de educação física - FEF/UFG

** Mestre, Professor da Faculdade de Educação Física - UFG

*** Doutor, Professor da Faculdade de Educação Física - UFG 
(OKUMA,1998).

Ingram (2000, apud MATSUDO et al. 2001) defende, com bases biológicas, que a diminuição do nível de atividade física com a idade acontece em conseqüência de uma liberação reduzida do neurotransmissor dopamina ou da perda dos receptores de dopamina, associados a alterações na locomoção. São os mesmos neurotransmissores que reduzidos na substância negra irão provocar os distúrbios motores e outros sintomas da doença de parkinson.

Desde a descoberta da doença de parkinson em 1817, várias estratégias de tratamento vêm sendo utilizadas a fim de minimizar ou até mesmo melhorar o quadro de sintomas que acomete o parkinsoniano, tais como: medidas farmacológicas, não farmacológicas e cirúrgicas. Grandes avanços foram obtidos nos tratamentos farmacológicos e, com grau variável de sucesso, nos tratamentos cirúrgicos. No entanto, o resultado dos tratamentos não farmacológicos depende de uma série de hábitos e medidas como educação, intervenção de suporte, nutrição e exercício físico, que devem ser tomados pelo parkinsoniano e profissionais de áreas afins, a fim de minimizar algumas de suas complicações. Porém, não atenua a gravidade da doença ou impede sua progressão, entretanto, mantém a pessoa melhor preparada para enfrentar as alterações orgânicas e psicológicas decorrentes do enfraquecimento muscular e acometimento motor típicos desta doença.

Sendo assim, a atividade física é parte fundamental na preservação e melhoria das funções motoras e nas relações com o bem-estar psicológico, comumente indicado por sentimentos de satisfação, felicidade e envolvimento do idoso, acometido ou não da doença. Confirmando esta teoria, Bromley (1990 apud OKUMA, 1998, p.83) afirma que "as alterações psíquicas e sociais que acompanham o processo do envelhecimento têm como uma de suas bases as alterações que se operam no nível biofísico". Então, qualquer intervenção que promova a expectativa de vida em todos os aspectos depende muito da melhoria dos aspectos biológicos sobre os psicológicos das pessoas idosas.

Um fator importante para o bem-estar psicológico e para a qualidade de vida do idoso é a manutenção de sua independência decidir por si só - e sua autonomia - competência para o domínio do ambiente, para realização das atividades da vida diária e das atividades instrumentais da vida diária, bem como reconhecer e lidar com o próprio 
corpo e com seu processo de envelhecimento e doença (OKUMA,1998).

Observa-se uma relação significativa do profissional de fisioterapia na prevenção e melhoria de dores musculares e articulares, anormalidades posturais e outras complicações trazidas pela imobilidade excessiva, com a aplicação de exercícios físicos ${ }^{3}$, mas, nada consta sobre a intervenção de um professor de educação física, na atuação interdisciplinar com outros profissionais de áreas afins, na integração dos vários saberes para a obtenção do reconhecimento na melhoria física, psíquica e existencial do Ser parkinsoniano.

Considerando que poucos estudos abordam os efeitos de um programa regular de atividade física generalizada, isto é, sem treinamento específico sobre os distúrbios motores na doença de parkinson, pensamos ser relevante analisar e utilizar, para alcançar a melhoria das capacidades físicas e emocionais de uma idosa parkinsoniana, um programa de atividades físicas relacionado à saúde, por oferecer certas vantagens. Uma dessas vantagens é proporcionar ao aluno uma variedade de rotinas motoras, apresentando diversas opções em termos de tarefas motoras, oportunizando não apenas a diminuição da monotonia de uma mesma rotina semana após semana, mas, sobretudo, aumentando a probabilidade de atender às suas diferenças individuais (GUEDES \& GUEDES, 1993).

A doença de parkinson é uma doença crônica, degenerativa e progressiva, que ocorre pela perda de neurônios no sistema nervoso central (SNC) em uma região conhecida como substância negra (ROWLAND,1986). A doença de parkinson acomete uma em cada cem pessoas, normalmente com mais de cinquenta anos: é a segunda doença neurodegenerativa mais comum (BRITO,1998).

Os sintomas da doença ocorrem devido à degeneração dos neurônios da substância negra; no entanto, o motivo que leva a essa degeneração é desconhecido.

Os distúrbios motores primários pertencentes a uma tétrade clássica são: tremor, rigidez muscular, bradicinesia e distúrbios posturais (ANDRADE, 2000). O tremor costuma ser bastante peculiar e característico. Geralmente é o primeiro a ser notado pela pessoa. Acomete primeiramente um dos lados do corpo, iniciando-se no membro superior (antebraço e mão), ou no membro inferior (pé). Com a evolução da doença, atinge o outro lado e freqüentemente se mantém assimétrico. $\mathrm{O}$ tremor pode ser comparado com o movimento de prono-supinação da 
mão, na forma de "contar dinheiro" ou "enrolar pílulas" com os dedos das mãos, ou de flexo-extensão do antebraço (ANDRADE, 2000).

A bradicinesia se manifesta por uma escassez de movimentos automáticos, como balançar os braços durante a marcha (ANDRADE, 2000).

A rigidez muscular ocorre porque os músculos não recebem sinais para relaxar, podendo assim, causar dores musculares e alterações na postura.

O encurtamento anterior gradual da coluna dorsal, a manutenção de semiflexão dos membros superiores e as perturbações dos reflexos posturais, provocam dificuldades para a recuperação do equilíbrio quando a pessoa se desloca (ANDRADE, 2000).

Sintoma que merece destaque é a depressão, pois, na doença de parkinson, ela ocorre cedo em sua evolução, mesmo antes dos distúrbios motores serem notados. Seu surgimento está relacionado com a redução do neurotransmissor serotonina, que provoca alterações emocionais, como sentimento de medo e insegurança diante da evolução da doença.

Outros sintomas podem aparecer à medida que a doença progride, porém variam de pessoa para pessoa. Algumas podem apresentar sintomas graves. Em geral, os sintomas mudam com o passar do tempo, afetando também a memória e o raciocínio.

Todos esses sintomas da doença, associados aos efeitos do envelhecimento na capacidade funcional, provocam um quadro de limitações físicas, incapacitando o idoso a realizar as atividades cotidianas, como caminhar alguns quarteirões, fazer compras e atividades que oferecem prazer. "Isto, por sua vez, fatalmente tem reflexos nos domínios sociais e psicológicos" (OKUMA,1998).

Porém, para que um programa de atividade física contribua para a melhoria de vida do idoso, mantendo-o ativo e participativo socialmente, os profissionais de educação física e áreas correlatas devem primeiramente entender o que é o ser idoso e suas estruturas existenciais a fim de que sua ação pedagógica aponte as necessidades reais e pessoais do indivíduo.

Segundo Okuma (1998), um ponto fundamental para o desenvolvimento de comportamento e atitude positiva em face da atividade física é respeitar as experiências humanas, compreendendo que em cada indivíduo há algo especial relacionado ao contexto pessoal 
de vida que pode estimulá-lo ou não a essa prática.

O programa de atividade física realizado neste estudo primou pelo respeito às diferenças individuais da aluna, por sua aceitação como legítima na convivência, e de seu ser no mundo com o seu corpo, num determinado espaço de atividades e num tempo propício, que contribuiu para a melhoria de suas capacidades físicas e qualidade de vida.

Este trabalho é parte de uma monografia de graduação apresentada como requisito para a conclusão do curso de licenciatura em educação física da Faculdade de Educação Física, Universidade Federal de Goiás.

\section{METODOLOGIA}

\section{Sujeito}

A investigação envolveu uma pessoa idosa de 69 anos, do sexo feminino (neste trabalho identificada com o nome fictício de Dona Lúcia), viúva há onze anos e que há dez anos identificou os primeiros sintomas da doença de parkinson.

\section{Local}

O estudo foi realizado no Setor União, em Goiânia, na residência da aluna e no Centro Goiano de Neurologia e Neurocirurgia, situado na rua 83 , no 133 , Setor Sul.

\section{Procedimentos}

Antes de iniciar as atividades, foi realizada uma avaliação médica prévia, e o sujeito considerado apto a desenvolver as atividades desse programa.

\section{Técnicas de Observação e Registro}

A coleta de dados foi realizada por meio de observações sustentadas e registradas (guiadas por um corpo de conhecimento), entrevistas não-estruturada e semi-estruturada e memorial descritivo.

Fazendo uso de um roteiro de observações, os observadores utilizaram um teste de exame motor (BRITO,1998), o qual identifica o nível de capacidade do sujeito em realizar movimentos comprometidos pela doença, registrando a capacidade da aluna para se levantar da 
cadeira, para caminhar, para executar movimentos de amplitude, de mobilidade e estabilidade.

Foram usados dois tipos de entrevistas: a primeira entrevista nãoestruturada, por permitir a realização de explorações e coletar dados na tentativa de obter maiores informações a respeito da entrevistada, registrando os dados relevantes e pertinentes ao estudo em questão; a segunda, na fase final do programa, semi-estruturada, a fim de avaliar a opinião do sujeito da pesquisa referente ao programa.

Como complementação, fez-se uso de um memorial descritivo, por se referir aos fenômenos da consciência, isto é, retrata sempre as formas de pensar de um indivíduo diante de situações vivenciadas. O conteúdo de um memorial diz respeito a emoções, crenças, valores, ansiedades, medos, contradições, prazeres e desprazeres do indivíduo (NEGRINE,1999).

Os investigadores utilizaram os relatos orais da aluna, registrandoos nos relatórios diários, como fonte documental, após as atividades, para auxiliar a discussão dos resultados.

\section{Protocolo de Avaliação}

A situação de teste foi observada e registrada durante todo o tempo nas diferentes fases do estudo pelos observadores participantes. Foram realizadas duas avaliações: no início e aos seis meses de aplicação do programa.

Para mensuração da capacidade de se levantar da cadeira, caminhar, executar movimentos de grande amplitude, movimentos que requerem certo grau de mobilidade e estabilidade postural, inerentes à manutenção da capacidade funcional, utilizou-se um teste de exame motor (BRITO, 1998).

O teste, em cada variável, consiste em avaliar de zero a quatro o nível de capacidade em executar o movimento da melhor forma possível, obtendo total controle do sujeito:

\section{I-Levantando-se da Cadeira}

(O paciente tenta levantar-se da cadeira com encosto reto, com os braços cruzados sobre o peito).
0- Normal
1- $\quad$ Lento ou necessita mais de uma tentativa 
2- $\quad$ Auxilia-se empurrando os braços da cadeira

3- $\quad$ Tende a cair para trás e pode necessitar de mais de uma tentativa, porém consegue sem auxílio

4- Incapaz de levantar-se sem auxílio

\section{II-Marcha}

0- Normal

1- Anda lentamente, pode arrastar os pés com pequenos passos, ausência de propulsão

2- $\quad$ Anda com dificuldade, precisa de pouco ou de nenhum auxílio; pode ter passos pequenos ou ausência de propulsão

3- Severo distúrbio de marcha, necessita auxílio

4- Não pode caminhar mesmo com auxílio

\section{III-Rigidez}

(Avaliada na movimentação passiva das grandes articulações com o paciente sentado e relaxado)

$0-\quad$ Ausente

1- Leve ou dectada apenas quando sob manobras de ativação

2- $\quad$ Leve a moderada

3- Acentuada, porém consegue-se o movimento em toda sua amplitude com facilidade

4- $\quad$ Severa dificuldade em obter o movimento em toda sua amplitude

\section{IV-Bradicinesia e Hipocinesia corporal}

(Combina lentidão, hesitância, diminuição do balanço dos braços, pequena amplitude e pobreza dos movimentos em geral)

0- Nenhum

1- Alentecimento (tornar-se lento, vagaroso) mínimo, dando ao movimento um caráter deliberado (pode ser normal para algumas pessoas). Possivelmente amplitude reduzida

2- $\quad$ Leve grau de alentecimento e pobreza do movimento, que é definitivamente anormal, alternativamente 
alguma redução de amplitude

3- Moderado alentecimento, pobreza ou amplitude do movimento

4- Alentecimento acentuado ou pequena amplitude do movimento

\section{V-Estabilidade Postural}

(Resposta ao súbito deslocamento posterior produzido por impulso nos ombros, enquanto o paciente ereto, olhos abertos e pés levementeseparados. Paciente preparado)

0 - Normal

1- Retropulsão, mas recupera-se sem auxílio

2- Ausência de resposta postural: pode cair se não seguro pelo examinador

3- Muito instável, tende a perder equilíbrio espontaneamente

4- Incapaz de permanecer em pé sem auxílio

\section{Programa de Atividade Física Generalizada}

Trata-se de um programa de educação física, cuja característica fundamental é ser educacional, ao visar dar ao sujeito com a doença condições para se autocuidar. O programa de atividade física generalizada tem como objetivo levar o aluno a: 1) aprender sobre a importância da atividade física no processo de evolução da doença; 2) perceber quais atividades físicas são mais adequadas para si e como realizá-las; 3) melhorar sua capacidade funcional e aptidão física e suas condições de saúde física e mental; 4) transferir para o cotidiano os novos conhecimentos, integrando-os à sua vida; 5) melhorar sua qualidade de vida.

As investigações desse programa se constituíram de conversas informais sobre os itens mencionados acima e atividades práticas, desenvolvidas em três sessões semanais de sessenta minutos, durante seis meses. O programa continua sendo desenvolvido mesmo após esse tempo, com novos efeitos que não serão mencionados neste presente trabalho.

Todos esses objetivos estão relacionados diretamente à melhoria nos distúrbios motores que acomete a pessoa com a doença de parkinson.

O conteúdo desenvolvido nas aulas práticas foi o seguinte: 
1) Atividades de alongamento ativo e passivo realizadas na posição horizontal ou vertical;

2) Atividades de relaxamento, massagem;

3) Atividades de expressões faciais, que levam o sujeito a perceber a ausência de movimentos involuntários automáticos, gradualmente abolidos durante a evolução da doença. Exemplo: as pálpebras ficam indolentes, piscando cada vez menos;

4) Atividades aeróbias, realizadas mediante caminhadas em diferentes ambientes;

5) Atividades respiratórias realizadas por meio de exercícios respiratórios;

6) Atividades para melhorar a força dos músculos dos membros superiores e inferiores, desenvolvidas por meio de exercícios tradicionais de ginástica, utilizando objetos como bola, bastão, halteres de meio quilo e atividades recreativas, como cabo de guerra, bocha adaptada com almofada e outras;

7) Atividades para melhorar o controle motor (agilidade, tempo de reação e de movimento e equilíbrio) realizadas por meio de habilidades motoras básicas, como manipular objetos (lançar, pegar e bater em bolas de diferentes tamanhos, balões e manipular bastões); locomover-se em situações e maneiras variadas (de lado, de frente, de costas, ultrapassando obstáculos, em cima de um risco, dentro de quadrados, subidas e descidas); equilibrar-se de distintas maneiras e situações (desde manterse e deslocar-se no nível do chão em superfícies baixas até chegar a superfícies mais altas), além de atividades rítmicas (com ou sem música) e dança;

8) Jogos recreativos que estimulem a memória, raciocínio e ofereçam prazer. Exemplo: jogo da memória (de figuras) e pontinho;

9) Trabalhos manuais com argila, pintura com giz de cera e colagem. Esse conjunto de atividades também busca levar ao resgate e ao reconhecimento das potencialidades físicas e motoras, bem como ao prazer de dominar e usar o corpo em situações de desafios.

\section{RESULTADOS E DISCUSSÃO}

Na Tabela 1 é mostrado o resultado da primeira avaliação do exame motor realizada com a Dona $\operatorname{Lúcia}^{3}$ antes de iniciar o programa 
de atividade física generalizada, na qual verificou-se que: 1) a aluna na primeira tentativa de se levantar da cadeira, com os braços cruzados sobre o peito, não conseguiu executar o movimento. Na segunda vez, levantou levemente o quadril do assento, porém caiu para trás. Só conseguiu se levantar quando apoiou os dois braços no assento da cadeira; 2) para analisar a marcha da aluna foi solicitado que Dona Lúcia desse a volta no quarteirão onde situa sua casa. Ao iniciar a caminhada, a aluna já se apoiou na professora. Não foi possível completar a atividade, devido ao cansaço que a aluna sentiu. Na caminhada observou-se lentidão nas passadas, arraste dos dois pés, principalmente o esquerdo, braço esquerdo imóvel e leve balanceio do direito, e postura moderadamente curvada; 3) quando solicitado movimento de amplitude como abrir os braços na lateral, ou levantar os braços à frente do corpo, observou-se limitações moderadas nos membros superiores. Nos membros inferiores, quando solicitado que elevasse o joelho, a aluna levantou levemente o pé do chão, não conseguindo elevar o joelho.

Nas situações em que se provocou desafio, foi verificado um elevado nível de tremor da mandíbula e dos braços.

Desse modo, alguns dos movimentos considerados essenciais para a realização das atividades instrumentais e da vida diária, tais como caminhar, levantar-se, vestir-se, cozinhar, etc, quando foram solicitados, aconteciam de forma bem limitada, podendo comprometer a capacidade de realizar tais atividades.

No início do programa, a aluna sentia sérias dificuldades motoras de coordenação, equilíbrio, amplitude de movimento, flexibilidade e aumento do tremor quando realizava movimentos que necessitassem levantar o pé do chão, como dançar, brincar de balão ou de bola, chutandoa ou batendo-a com a mão e brincadeiras que necessitassem agilidade; porém, sentia-se satisfeita em estar experimentando novos movimentos. De acordo com Silva et al. (2002), a atividade física generalizada tem a "intenção de poder atender melhor as necessidades do dia-a-dia dos idosos", pois possibilita o exercício de várias habilidades e capacidades físicas.

Após dois meses do programa, segundo Dona Lúcia, as dores na coluna e nas pernas haviam diminuído. Caminhar distâncias maiores que um quarteirão já era possível, porém a aluna continuava arrastando o pé esquerdo. Sentia moderado cansaço ao caminhar necessitando parar 
para se restabelecer. Já era capaz de levantar-se da cama sem ajuda, entretanto, demorava em se levantar, necessitando de mais de uma tentativa.

No quarto mês, a aluna relatou que estava tendo mais facilidade para pentear seu cabelo, porque antes sentia dores, cansaço e dificuldade para levantar o braço, caracterizando fraqueza muscular e rigidez das articulações. No último mês, já estávamos caminhando não só no bairro dela, mas indo até o bairro onde mora seu filho que corresponde a uns 15 quarteirões de sua casa, uma caminhada de cerca de quarenta e cinco minutos.

Outras avaliações da capacidade funcional foram realizadas durante a pesquisa, porém não serão analisadas neste estudo. Entretanto, achamos pertinente relatá-las, pois o objetivo do programa era ir ao encontro das expectativas da aluna. Em uma dessas avaliações, verificou-se que a força de Dona Lúcia para apreensão de objetos estava relativamente fraca. Diante de outras melhoras, notou-se que a aluna continuava sentindo fraqueza e dificuldade em fechar torneiras, abotoar roupas e outras atividades de apreensão. Diante de tal fato, re-elaborou-se algumas atividades que enfatizavam o trabalho com as mãos como cabo de guerra, argila, colagem, apertar bolinhas de diferentes tamanhos etc.

Tais atividades contribuíram para atingir os objetivos citados anteriormente, nos quais a atividade prática tem como meta levar o sujeito a produzir conhecimentos e se conscientizar de seu corpo, de suas capacidades funcionais, de suas habilidades motoras e de suas limitações, a fim de construir, junto com o professor, novos direcionamentos para as atividades propostas.

$\mathrm{Na}$ Tabela 2, observa-se que houve uma melhora geral nas capacidades motoras da aluna entre a primeira e a segunda avaliação, porém em níveis diferentes. Estas diferenças podem estar associadas, primeiramente, à variação dos níveis encontrados no primeiro exame motor realizado e, posteriormente, à ênfase que o programa pode ter oferecido para algumas incapacidades em detrimento de outras, mesmo considerando que todas as capacidades motoras são relevantes.

Uma outra avaliação foi realizada no Centro Goiano de Neurologia e Neurocirurgia pela neurologista, constatando que após seis meses de um Programa Regular de Atividade Física Generalizada a paciente "melhorou muito sua capacidade de movimentação, e houve melhora do equilíbrio, no tônus e na rigidez". 
As alterações emocionais, como a depressão, diminuíram, fato constatado pela médica na última avaliação realizada, a qual retirou um medicamento antidepressivo do seu coquetel de medicamentos anti-parkinsoniano. Contudo, essa diminuição não se mantém constante: oscila provocando falta de disposição para fazer as aulas, chegando até mesmo a provocar na aluna descrédito pelo programa.

Todas essas oscilações recebiam uma atenção e cuidados redobrados. Eram alterados ou adaptados, sempre que necessário, os objetivos da aula proposta em dias nos quais a aluna se encontrava chateada. Exemplo: aproveitar para caminhar até uma praça, sentar e conversar.

O programa de atividade física, juntamente com o papel do professor e da equipe, contribuiu muito para a melhora da aluna, porém nada somos sem o apoio da família e seríamos muito mais com a sua participação, unindo forças a favor de reafirmar a importância do ser idoso na família e na sociedade.

TABELA 1 Resultado da $1^{\text {a }}$ avaliação do exame motor ${ }^{*}$

\begin{tabular}{|l|r|r|r|r|r|}
\hline NÍVEL $1^{\mathrm{a}}$ AVALIAC̃̃̃O \\
\hline EXAMEMOTOR & 0 & 1 & 2 & 3 & 4 \\
\hline LEVANTARDACADEIRA & & & & $\mathrm{X}$ & \\
\hline MARCHA & & & $\mathrm{X}$ & & \\
\hline RIGIDEZ & & & & & $\mathrm{X}$ \\
\hline BRADICINESIAE HIPOCINESIACORPORAL & $\mathrm{X}$ & & & & \\
\hline ESTABILIDADE POSTURAL & & & & $\mathrm{X}$ & \\
\hline
\end{tabular}

* Fonte: BOLETIM DA DOENÇA DE PARKINSON. Coordenação de Marco Brito "Aprendendo a conviver com o mal de Parkinson" Revista Momento - (on line) Jan/Fev 1998. Acesso em:04 nov.2003.

TABELA 2 Resultado da $2^{\text {a }}$ avaliação do exame motor*

\begin{tabular}{|l|c|c|c|c|c|}
\hline NIVEL & \\
EXAMEMOTOR AVALIAÇÃO & & & & & \\
\hline LEVANTARDACADEIRA & 0 & 1 & 2 & 3 & 4 \\
\hline MARCHA & $\mathrm{X}$ & & & & \\
\hline RIGIDEZ & & $\mathrm{X}$ & & & \\
\hline BRADICINESIAE HIPOCINESIA CORPORAL & & $\mathrm{X}$ & $\mathrm{X}$ & & \\
\hline ESTABILIDADEPOSTURAL & & $\mathrm{X}$ & & & \\
\hline
\end{tabular}

*Fonte: BOLETIM DA DOENÇA DE PARKINSON. Coordenação de Marco Brito “Aprendendo a conviver com o mal de Parkinson" Revista Momento- (on line) Jan/Fev 1998. Acesso em: 04 nov.2003. 


\section{ENTREVISTAS}

Concluída a parte das observações do estudo, num segundo momento, logo após o término das avaliações, fez-se uso de uma entrevista semi-estruturada para se avaliar a opinião do sujeito da pesquisa, ou seja, queríamos saber: 1) como a aluna se sentia antes e após a aplicação do Programa de Atividade Física Generalizada; 2) se o programa preencheu suas expectativas; 3 ) como a aluna classificava o programa. Abaixo teremos uma visão panorâmica das respostas do sujeito.

Diante das respostas da aluna, feita a primeira pergunta, Dona Lúcia respondeu que antes ela não conseguia levantar da cama sozinha, hoje consegue; ela vai à casa do filho e não cai mais durante o percurso; ela vai andar e lembra o que a professora falou: tem que levantar o corpo, observar onde pisa, balançar os dois braços.

Quando se perguntou se o programa preencheu suas expectativas, Dona Lúcia respondeu que sim, porém achou que iria ficar melhor, mais rápido "porque melhora uma coisa e piora outra"; "eu sei que não vou sarar", "mas às vezes melhora", "tá, bom!".

Dona Lúcia classificou o programa como muito bom. Quando perguntou-se por que, ela respondeu: "porque eu gosto", "gosto de você", "de fazer muitas coisas que eu não fazia".

\section{CONCLUSÃO}

De acordo com os resultados do estudo, pode-se concluir que um Programa Regular de Atividade Física Generalizada, portanto não específico para qualquer capacidade física ou habilidade motora, desenvolvido por um curto período de tempo ${ }^{5}$ e direcionado para uma pessoa idosa com a doença de parkinson, é capaz de melhorar as capacidades físicas desta com o passar de dois meses, elevando o nível de flexibilidade, agilidade, marcha e equilíbrio. Nota-se nas tabelas de avaliações que os distúrbios motores ainda persistem, porém em níveis mais baixos e só saberemos o quanto esses níveis podem abaixar se continuarmos com o programa.

Tal conclusão é de extrema importância para pessoas idosas com a doença de parkinson, uma vez que a melhoria dessas capacidades físicas contribuiu parcialmente para a manutenção da autonomia nas 
atividades da vida diária e nas atividades instrumentais da vida diária. A respeito das alterações emocionais podemos concluir que estas ainda persistem. Porém, com a ajuda da ação pedagógica do professor, o sujeito partiu para a descoberta de si, ao autoconhecimento, a fim de que possa sempre atualizar suas potencialidades e reconhecer-se como singular. Ao refletir sobre a experiência, tal pesquisa levou-nos à melhor compreensão do Ser idoso e suas implicações. Contudo, para entendêlo foi preciso mergulhar na vivência do sujeito, em busca de uma mudança em sua estrutura existencial. Este mergulho possibilitou vislumbrar um novo horizonte em trabalhos desta natureza.

A relevância da pesquisa foi demonstrada não só pelas melhorias nas capacidades físicas da aluna, mas pelo fato de a mesma ter considerado este trabalho de extrema importância.

The Effects of a Regular Physical Activity Program on the Motor Disorders of a Parkinson Patient.

\begin{abstract}
This study aims at analyzing the effects of a six-month program of regular physical activity upon the motor disorders of an elderly Parkinson patient. The subject was an elderly, physically inactive woman, who has had Parkinson for ten years. The six-month program was developed with the help of the Centro Goiano de Neurologia e Neurocirurgia (The Goianian Center for Neurology and Neuro-surgery). A motor exam (Brito, 1998) was used to evaluate the level of rigidness, bradicinesia, the capacity to raise from a chair and walk, and posture changes. Evaluations were conducted before and after the program. After two months of regular physical activity, improvements were noticed on rigidness, bradicinesia, and balance, which brought autonomy and independence to the subject. KEY-WORDS: Parkinson - elderly - physical activity

Efectos de un programa regular de actividad generalizada sobre los disturbios motores de una persona con enfermedad de parkinson RESUMEN
\end{abstract}

El objetivo de este estudio fue analizar los efectos de seis meses de un programa regular de actividad física sobre los disturbios motores en una persona mayor con la enfermedad de parkinson. El sujeto investigado fue una persona mayor del sexo femenino, sedentaria portadora de la enfermedad hace diez años. El programa fue desarrollado durante seis meses con el apoyo del Centro Goiano de Neurología y Neurocirugía. Para evaluar el nivel de rigidez, la bradicinesia, la capacidad de levantarse de la silla y locomoverse y las alteraciones posturales fue utilizado un examen motor (BRITO, 1998), con evaluaciones antes y después del programa. Con dos meses de actividad física hubo mejora en la 
rigidez, en la bradicinesia y en el equilibrio, contribuyendo para la autonomía e independencia del sujeto investigado.

PALABRAS CLAVES: enfermedad de parkinson - persona mayor - actividad física

\section{NOTAS}

${ }^{1}$ Ueno (1999, apud AMORIM et al., 2002, p.51) define capacidade funcional como uma autonomia física, traduzindo-se numa independência para realizar as atividades de vida diária, tais como atividades de deslocamento, de autocuidado, ocupacionais e recreativas.

${ }^{2}$ Segundo Nahas (2001) define-se exercício físico como uma das formas de atividade física planejada, estruturada, repetitiva, que objetiva o desenvolvimento da aptidão física, de habilidades motoras ou a reabilitação orgânico-funcional.

${ }^{3} \mathrm{O}$ nome da aluna é fictício para preservar sua integridade.

${ }^{4}$ Nota-se aqui a importância do trabalho interdisciplinar de uma equipe em que a psicologia poderia ajudar.

${ }^{5}$ Logo, se o tempo de doença é de 11 anos, 6 meses é um curto período.

\section{REFERÊNCIAS}

ANDRADE, Luiz Augusto Franco de. Doença de parkinson. In: SOUZA, Sebastião Eurico de Melo (Org.). Tratamento das doenças neurológicas. Rio de Janeiro, RJ: Guanabara Koogan,2000.

ANDRÉ, Marli Elizza Dalmazo Afonso de. Etnografia da prática escolar. Campinas, SP: Papirus, 1995.

AMORIM, Paulo et. al. Estilo de vida ativo ou sedentário: impacto sobre a capacidade funcional. Revista Brasileira de Ciência do Esporte, Campinas, v. 23, n. 3, p. 49-63, mai. 2002.

BRITO, Marco. Boletim da doença de Parkinson. Aprendendo a conviver com o mal de Parkinson. Revista Momento (on line) Jan./Fev. 1998. Disponível em: <(mabrito@ doencadeparkinson.com.br)>. Acesso em: 04 nov. 2003. 
DUARTE, Cátia Pereira et al. A concepção de pessoas de meia-idade sobre saúde, envelhecimento e atividade física como motivação para comportamentos ativos. Revista Brasileira de Ciência do Esporte, Campinas, v. 23, n. 3, p. 35-48, mai. 2002.

GIL, Antônio Carlos. Método e técnicas de pesquisa social. 5. ed. São Paulo: Atlas, 1999.

GUEDES, Dartagnan Pinto; GUEDES, Joana Elisabete Ribeiro Pinto. Subsídios para implementação de programas direcionados à promoção da saúde através da educação física escolar. Revista da Associação dos Professores de Educação Física de Londrina, Londrina, v. 7, n. 15, p. 3-11, 1993.

MATSUDO, Sandra M.; MATSUDO, Victor K. R.; NETO, Turíbio Leite Barro. Perfil do nível de atividade física e capacidade funcional de mulheres maiores de 50 anos de idade de acordo com a idade cronológica. Revista Atividade Física e Saúde, v. 6 , n. 1, p. 12-24, 2001.

MOLINA, Rosane Maria Kreusburg.. O enfoque teórico metodológico qualitativo e o estudo de caso: uma reflexão introdutória. In: NETO, Vicente Molina, TRIVIÑOS, Augusto N. S. (Org.). A pesquisa qualitativa na educação física: alternativa metodológica. Porto Alegre: Universidade/UFRGS/Sulina, 1999.

NAHAS, Markus Vinicius. Atividade física, saúde e qualidade de vida: Conceitos e Sugestões para um estilo de vida ativo. Londrina: Medgraf, 2001.

NEGRINE, Airton. Instrumentos de coleta de informações na pesquisa qualitativa. In: NETO, Vicente Molina; TRIVIÑOS, Augusto N. S. (Org.). A pesquisa qualitativa na educação física: alternativa metodológica. Porto Alegre: UFRGS/Sulina, 1999.

NETO, Francisco Luiz de Marchi. Apostila de ginástica II Educação Física, Goiânia: UFG, 2002. 
MOLINA NETO, Vicente, TRIVIÑOS, Augusto N. S. (Org). A pesquisa qualitativa na educação física: alternativa metodológica. Porto Alegre: /UFRGS/Sulina, 1999.

OLIVEIRA, Marta Kohl. Vygotsky- Aprendizado e desenvolvimento um processo sócio- histórico. 3. ed. São Paulo: Spicione,1995.

OKUMA, Silene S. $O$ idoso e atividade física. São Paulo: Papirus, 1998.

ROWLAND, Lewis P. Merritt (Tratado de Neurologia). Rio de Janeiro: Guanabara Koogan, 1986.

SEVERINO, Antônio Joaquim. Metodologia do trabalho científico. São Paulo: Cortez, 1993.

SILVA, Veronica Miyasike da; VILLAR, Rodrigo; ZAGO, Anderson Saranz. Nível de agilidade em um indivíduo entre 42 e 73 anos: Efeito de um programa de atividades físicas generalizadas de intensidade moderada. Revista Brasileira de Ciência do Esporte, Campinas, v. 23, n. 3, p. 35-48, mai. 2002.

WERNECH, Hamilton. A sabedoria está na simplicidade. Petrópolis, RJ: Vozes, 2003.

FRONTERA, Walter R., D. Dawson e D. Slovik. Exercício físico e reabilitação. Porto Alegre: Artemed Editora, 2001.

WEINECK, Jürgen. Treinamento Ideal.. São Paulo: Editora Manole,1999.

Recebido em: novembro de 2003. Aprovado em: dezembro de 2003.

Endereço para correspondência:

Marcus Fraga Vieira e-mail: Marcus@fef.ufg.br

Universidade Federal de Goiás - Faculdade de Educação Física

Campus II - Goiânia-GO

Pensar a Prática 7(1): 27-43, Mar. - 2004

CX. Postal 131 - 74001-9793 
